\title{
Water Safety Plan implementation in East Africa: motivations
}

\section{and barriers}

Alison Parker $^{1 *}$; Corinna Summerill ${ }^{1}$

1. Cranfield Water Science Institute., Cranfield University, Cranfield, Bedfordshire MK43

0AL, +44 1234 75011, a.parker@cranfield.ac.uk

Alison Parker is the Academic Fellow in International Water and Sanitation at Cranfield

University. Corinna Summerill completed her PhD at Cranfield University

\begin{abstract}
Water Safety Plans (WSPs) are a comprehensive source to tap risk management approach that are recognised as the most effective way of ensuring drinking water safety. In East Africa there is only one utility implementing them, so this research aims to identify barriers for WSP implementation in this region, as well as potential motivating factors. This is achieved through twenty semi-structured interviews with utilities, regulators and international agencies. The biggest motivating factor was the potential of WSPs to improve standards and reduce water quality incidents, with the potential for cost savings and NRW reduction being further motivations. However, in many utilities senior managers are not motivated to start implementing WSPs and they can prevent other staff from doing so. Smaller or weaker utilities may struggle to implement a WSP as they are constantly engaged in their day-to-day problems and do not have the capacity to plan in the longer term.
\end{abstract}

Keywords: Africa, organizational change, public health, risk management, water quality Water Safety Plan 


\section{Introduction}

The third edition of the WHO Guidelines for Drinking Water Quality (WHO 2004), complementary publications 'Water Safety Plans' (Davison et al. 2005) and more recently the WSP Manual (Bartram et al. 2009) advocated the use of WSPs, a comprehensive 'source to tap' risk management approach, as the most effective way of ensuring drinking water safety. The primary aim of WSPs is public health protection, involving system assessment, operational monitoring and management plans, which are guided by health-based targets and overseen by surveillance. The most up to date WSP guidance describes an eleven step approach (Figure 1). The WSP process should not be seen as a one off exercise, and the cyclical nature, involving review, approval and audit to ensure continuous improvement is emphasised. What is important is that the WSP guidance is flexible, as long as these steps are followed, there may be different methods used. For example risk ranking approaches may vary depending on the organisations current risk management practices. Risk assessment is 'not a goal in its own right' and should be seen as a tool to aid management decisions and assist in incremental improvements to water quality (Davison et al. 2005).

Since their promotion began in 2004, WSPs have been gaining momentum and ever increasing numbers of water utilities are implementing them (Summerill 2010). Public health protection is the main aim of a WSP, and therefore should be a main motivation. However, utilities are also motivated by the framework for improved procedures that WSPs offer for monitoring (Godfrey et al 2005; Dyck et al 2007, Gunnarsdottir and Gissurason 2008; Jayaratne 2008; Mälzer et al 2010; Viljoen, 2010) and the potential this brings for identifying and reducing non-revenue water (NRW) (Dyck et al 2007; Summerill et al 2010). Utilities also want to improve their image and accountability and prevent the recurrence of 
past water quality incidents (Dyck et al 2007; Gunnarsdottir and Gissurason, 2008; Summerill et al 2010).

Some regulators have introduced legal requirements for WSP implementation, including, amongst others, the State Government of Victoria, Australia (Jayaratne 2008); Japan (Yokoi et al 2006); and the UK (Bartram et al. 2009) and WSPs provide independent verification of ISO 9001 (Viljoen 2010). However, existing regulations are sometimes perceived as insufficient to ensure water safety alone thus motivating utilities to implement WSPs as a way of achieving improved water safety (Summerill et al 2010).

The support from other stakeholders and the utilities' own staff (including managers at all levels) can be a driving factor for WSP implementation (Mahmud et al 2007; Viljoen, 2010), conversely a lack of co-operation from these groups can form a barrier (Gunnarsdottir and Gissurason 2008; Bartram et al 2009; Mälzer et al 2010; Summerill 2010, Summerill et al 2010). Some stakeholders, including the utilities themselves are simply unaware of the WSP approach (Summerill et al 2010). If water quality is consistently good then WSPs may not be considered urgent, and complacency occur (Bartram et al 2009; Mälzer et al, 2010).

A lack of resources for WSP implementation has been cited as a challenge by service providers of all scales, from large serving millions of consumers to caretakers of community supplies in Bangladesh (Mahmud et al 2007; Gunnarsdottir and Gissurason 2008; Bartram et al 2009; Summerill et al 2010). However, Godfrey et al (2005) concluded from their experience in Guntur, Andhra Pradesh, India that insufficient data does not limit the development of WSPs. Summerill (2010) warns that a lack of resources can sometimes be used as an 'excuse' for not implementing WSPs, and even a basic WSP done with what resources are available is better than no WSP at all. 
These motivations and barriers will impact the scale of WSP implementation. In East Africa WSPs have only by one utility, National Water and Sewerage Corporation, in two of the cities it serves in Uganda, Kampala and Jinja (Tibatemwa 2005). This research thus aims to identify what the barriers are to further WSP implementation in East Africa, and what the motivating factors might be. The five countries focussed on in East Africa were Kenya; Tanzania; Rwanda; Uganda and Ethiopia.

\section{Methodology}

In order to generate the richness of data required, in-depth qualitative interviewing was adopted as the chosen methodology, as opposed to, for example more quantitative techniques such as questionnaires (Rubin and Rubin 1995). Interviews were conducted with twenty key informants. The respondents are listed in table 1 by type, though their anonymity has been preserved in order to comply with Cranfield University's ethical guidelines. In order to fully understand the implications of their responses, they have been categorised into three types, "international agencies" which include any organisation that engages internationally on WSPs, "water governance entities" (a broad term to describe other national stakeholders who are involved in water governance) and "utilities".

Potential interviewees were identified from lists of contacts provided by the International Water Association (IWA) and Water Operators Partnerships (WOPs). Only a small proportion of utilities contacted responded, and it is probable that these utilities are the most advanced in the region with regards to WSP implementation. As such, their responses should not be taken as perfectly representative of utilities in the region. Utilities were also able to nominate any staff member to complete the interview. Five of the respondents were middle managers with a particular responsibility for WSPs or water quality. Only three respondents were CEOs or Managing Directors, but these were from smaller utilities that were not yet 
developing WSPs, hence they were not able to talk in detail about their motivations and barriers for doing so. A number of international experts were also identified, all of whom had experience in East Africa.

Interviews were semi-structured. The questions were based on motivations and barriers documented in the literature. Approximately half were carried out by phone, the remainder in person. Notes were taken during the interviews. All but one of the interviewees spoke good English; the Rwandan supplier was interviewed in French with the translator providing notes afterwards. The main and secondary motivations and barriers and responses to yes/no questions were tallied on a spreadsheet. Other qualitative responses were grouped into categories.

Respondents were asked to name the main motivation and barrier for Water Safety Plan implementation, in their experience. They were also asked to name any further motivations and barriers, which are referred to throughout as "secondary motivations" or "secondary barriers". They were also asked what further support utilities require.

This study protocol was approved by Cranfield University's Science and Engineering Research Ethics Committee (number 88, 10/08/10).

\section{Results}

The initial section summarises the interview responses relating to WSP motivations, capturing both the qualitative and quantitative nature of the interviews.

Improved water quality The need to improve water quality and or/health was identified as the main motivating factor for WSP implementation. Improved standards and/or health were also named as a secondary motivating factor by three further utilities. One international agency said utilities are aware that people avoid drinking tap water if they can afford bottled water 
and a Kenyan supplier also cited this as a secondary motivating factor. Six respondents believed that WSPs could reduce water quality incidents, versus two who said there was insufficient evidence as yet.

Internationally recognised standards The ability of WSP to assist utilities gaining ISO certification was cited as a main or secondary motivation by only one two respondents. However all the utilities said it would be a motivating factor when asked specifically. The fact that WSP implementation does not result in an internationally recognised standard (unlike ISO 9001) may in fact be a barrier to implementation as it means that utilities may not take WSPs seriously. A step forward could be for the WHO or regulators to give more official certification to utilities implementing a WSP.

Cost savings Cost savings were the third most popular motivating factor, and were also named by four further respondents as a secondary motivating factor. When asked specifically eight utilities said it would be a motivating factor. Money can be saved by reducing monitoring and testing. Whilst one international agency said that there was so far insufficient evidence for cost savings as a result of implementing a WSP, the remainder thought that costs savings were inevitable, as did all the water governance entities. They cited examples of cost savings achieved in England, Wales, Brazil and Uganda.

Regulatory requirement As WSPs are not yet a regulatory requirement in East Africa this was not named as a motivating factor. One regulator cited the lack of policy supporting WSPs as the main barrier. When asked if WSPs should become a regulatory requirement, the international agencies thought that the regulators were not capable of enforcing WSP implementation. All the water governance entities asked in this survey and all but one of the utilities thought that they should be a regulatory requirement. The international agencies believed that regulators are weak and struggling with a lot of issues. One respondent believed 
that WSPs are not a priority in the medium term in most low income countries and governments should invest in resources and capacity building for WSPs. Many regulators are not even aware of WSPs, and so far international programmes have not addressed this. Weak regulation is preventing one utility from expanding its WSP programme into medium sized networks - they are trying to address targets that don't exist. However, if it were a regulatory requirement it would enhance top management support.

$N R W$ The importance that utilities put on NRW is demonstrated by the fact that in WOP's most recent call for proposals, the majority addressed NRW. However, it was not cited as a main motivation, and it was cited as a secondary motivation by only one utility. When asked specifically whether reduced NRW would motivate them to implement WSPs, seven out of ten responded that it would. Three international agencies and three regulators believed that WSPs can reduce NRW, although one thought that it would not be reduced and two thought that there was insufficient evidence. To build up the evidence for NRW reduction is key, since it is a priority for utilities. Indicators for NRW should be embedded in the WSP from the start.

Improved image and accountability Whilst all the utilities asked in this survey responded that their company's image was extremely important, one of the international agencies commented that some utilities do not care about their image to their customers (these utilities are also unlikely to respond to requests for interviews; hence their views are not represented here). One supplier stated that their desire to be viewed as a model supplier was the main motivating factor for WSP implementation. A regulator stated that increased accountability was the main motivating factor for WSP implementation. When asked specifically, eight utilities agreed that improved image and accountability were motivating factors for WSP implementation. 
The second section summarises the interview responses relating to WSP barriers

Lack of evidence The lack of evidence (or understanding) for the benefits of WSPs was cited as the main barrier by one international agency and a secondary barrier by another. A published list of benefits - including 'additional' benefits such as financial benefits, reduced NRW and an ability to prioritise investment, as well as an inventory of the costs and time demands of implementation may therefore increase motivation. A regulator said a secondary barrier was the lack of relevant examples for rural areas. However one utility cited examples of success as the main motivating factor for WSP implementation.

Inadequate control of resource protection Whilst most utilities have control over their treatment and distribution networks, often protection of water resources was out of their control. This was cited as the main barrier by two respondents. Two further respondents recognised the role of WSPs in sustainability and climate change preparedness as a secondary motivating factor. A regulator also said that water treatment costs were increasing because of deteriorating water quality. In some situations water resources might be under the control of other organisations who are not implementing a WSP. Cited examples include another municipal authority, the Wildlife Service and the Forestry Department. Communities also have a role to play in resource protection, as they may pollute the water source with fertiliser or settle in the wetlands where groundwater is recharged. This barrier can be overcome with effective stakeholder engagement and Integrated Water Resources Management.

Inadequate senior management support and overall staff commitment. Commitment from senior management was universally recognized as being an important component of WSP implementation. Senior management can ensure that all staff understand their role in WSPs. However one regulator identified it as the main barrier to WSP implementation, and three other respondents identified it as a secondary barrier. Senior management need to be 
receptive to the ideas of staff who have attended external training courses. However, if a sensitized CEO is replaced (some utilities had three or four CEOs a year) the WSP process can be halted. Senior management need to appoint a WSP officer to oversee the process; two utilities cited the lack of a dedicated officer as a secondary barrier. One utility and one international agency cited a lack of time or prioritization as the main barrier to WSP implementation, with two further respondents citing it as a secondary barrier. This barrier can be overcome by senior management who can ensure WSPs are prioritized over other activities and reduce the number of competing programmes. This applies for both any additional training programmes or workshops required by the WSP, and generating information that is required by the WSP team. In all other areas interviewees spoke in terms of institutional barriers, but this was one area where interviewees hinted that their opinions might differ from those of their senior managers. In both utilities that were more advanced in WSP implementation and those just starting to develop one there was frustration that senior managers did not support the process or did not encourage other staff to commit time to it. For example one utility received invitations for eight staff to attend the IWA Water Safety conference in Malaysia, but the CEO only let three chemists attend. Another middle manager reported that the individuals who had attended the training course were better motivated to implement WSPs and understood the process better than other staff. All the utilities asked were able to name an example of staff from different divisions working together on a project, so inter-department co-operation should not form a barrier to WSP implementation.

Inadequate training Five of the utilities believed they understood the steps required for a WSP. The three respondents who said they did not understand the steps were senior managers, although they were all from small utilities that had not yet started developing a WSP. Inadequate staff capacity was one of only two barriers cited by senior managers, and 
capacity building was their only request for support. Six of the utilities believed they had the skills required for a WSP, versus two who did not. All the utilities who requested further support to implement WSPs required additional training, and eight further respondents mentioned training or capacity building in some form. Lack of knowledge or skills was cited by the most respondents (five) as the main barrier to WSP implementation, and by five further respondents as a secondary barrier. An international agency thought that the management aspects were the biggest capacity gap.

Costs A lack of funds to implement WSPs was cited as the main barrier for four of the utilities. A further utility named the lack of resources as a secondary barrier. A lack of funds and resources was the one of only two barriers that was cited by the senior mangers interviewed. Six respondents requested that funds for control measures be included in support plans for utilities, and three suggested funds for monitoring equipment. However, regulators and international agencies did not cite a lack of funding as the main or secondary barrier. This suggests there need to be greater sensitisation of utilities to the wider economic benefits of WSPs. Utilities have only limited funds and WSPs are perceived as being extra work with no perceived pay off - so they need to be shown examples where cost savings have been demonstrated. This works on both a utility and a departmental scale - senior management need convincing that the WSPs will ultimately save money overall, but department managers also need to be persuaded to release money from their budgets for the required control measures. Budgets may need to be reallocated as whilst there are savings in water quality testing, inspection costs (e.g. transport costs) increase. Developing WSP tools may reduce the costs as utilities then do not need to employ expensive consultants to guide the process. It is also important to keep focussed so bureaucracy is minimised and hence costs do no increase.

Inadequate human resource Eleven respondents thought that utilities did have sufficient human resource to implement WSPs. No respondents cited it as a barrier. However, one 
utility respondent thought that some people in his company were misplaced and they need to do a job evaluation. With WSPs there is a shift in workload from the laboratory staff to the inspectors. This change needs to be incremental, as there is a big impact on the human resource. It is possible at this stage that even senior managers can end up out of a job.

Lack of priority of planning. All the utilities asked in this survey were in the process of implementing, or at least considering implementing a WSP. However, international agencies and water governance entities said that many utilities are so tied up in dealing with day-today challenges, such as leakage, they cannot make long term plans like a WSP. They struggle with political pressure and population growth. The challenge is to convince utilities to make a small start and not to stop because it is too complicated. All respondents were asked whether expanding the business or improving standards for current customers was important for utilities. The responses from international agencies and the utilities themselves were divided equally. However, the all water governance entities believed that utilities were more concerned with expanding their business.

Inadequate laboratory facilities Respondents were asked whether East African utilities had sufficient laboratory facilities to implement a WSP, and there was no consensus either way, although there was some concern that most of the smaller utilities would not have sufficient resource.

Insufficient documentation Whilst six of the nine utilities were convinced that the documentation of their systems was sufficient, none of the international agencies agreed. This may be because the utilities were unwilling to admit their inadequacies in the survey, or it may be because they do not understand the level of documentation that is actually required.

\section{Discussion}


East African utilities are motivated by the same factors as utilities worldwide to implement a WSP: improved image, improved accountability, reduced NRW, cost savings, qualification for other standards, buy-in of senior management and the support of external agencies. Conversely a low regard for image or inadequate senior management along with a lack of skilled staff, the perceived costs of implementing a WSP and the lack of awareness form barriers in East Africa and globally. In Uganda, suppliers learned that control measures identified by a WSP need to be simple or they will never be implemented (Tibetemwa 2005). To overcome this elsewhere, such as in Bangladesh and Iceland, appropriate or simplified tools have enabled operators (particularly small ones) to effectively implement WSPs (Mahmud et al 2008; Gunnarsdottir and Gissurason 2008), but these are yet to be introduced in East Africa. The process also needs to be explained clearly to the managers who will be releasing funds for the control measures to maintain their commitment to the WSP process.

Whilst Australia, Japan and the UK have introduced WSPs into their regulatory requirements (Jayaratne 2008; Yokoi et al 2006; Bartram et al. 2009), this has not yet happened in East Africa. In countries where there is no such support, some utilities treated this as a motivation as they believed the regulations are lacking and they want to comply with international standards. However, other utilities are discouraged, and this forms a barrier to implementation. Stakeholders were divided as to whether East African regulators had the capacity to enforce WSP implementation and whether engaging regulators should be a priority for external agencies. The fact that having implemented a WSP does not in itself qualify the supplier for any international mark or standard may also be a barrier.

In Bangladesh the ability of WSPs to involve all stakeholders was seen as a motivation (Mahmud et al 2008), although poor relationships with other stakeholders formed a barrier in several countries. In East Africa the latter case was true, and utilities were particularly 
concerned about the lack of co-operation from stakeholders involved in catchment management.

There were plenty of global examples of where poor relationships between staff had hindered WSP implementation (Gunnarsdottir and Gissurason 2008; Bartram et al 2009; Mälzer et al 2010; Summerill et al 2010). In East Africa, all the utilities reported that their staff were used to working well in teams together, and most blamed poor staff involvement in WSPs on the lack of direction from senior management rather than unwillingness in the staff members themselves. In Uganda, they found that the lowest level staff may hold the local knowledge which is essential for understanding the whole system from catchment to consumer (Tibetemwa 2005).

In order to increase WSP implementation in East Africa (and other developing regions), WSP promoters need to provide evidence for the benefits of WSPs. Not all stakeholders were aware of the evidence that WSPs reduce water quality incidents, costs and NRW. The evidence should be clearly presented to persuade utilities of the need for WSPs

Typically external programmes only train three staff from a supplier, whereas a WSP team can be larger than this, and implementation will involve all staff members. Programmes should seek to understand how messages are conveyed in a utility after a training event and how change management occurs. They should thus equip trainees to be the agents of change and to train their colleagues, as clearly most external agencies do not have the resources to train all staff. Programmes should also seek to engage with other external stakeholders, including:

- Larger associations, for example groups of utilities. These associations are engaged in information sharing so they just need to include WSPs at their events. 
- Donors and funding agencies. These can provide the required capital, and make WSPs a mandatory part of their programmes.

- Asset holding companies. Utilities may not have the capacity to invest in the necessary control measures.

- Regulators and politicians. They can then implement pro-WSP policies.

- Small operators in informal settlements and rural areas.

Although many stakeholders did not believe that costs were a barrier to WSP implementation; the utilities did. It is clear that even if there are overall cost savings, there needs to be some investment in the control measures in the early stages, which may be beyond the capacity of utilities that have poor cost recovery mechanisms.

The main limitation to the research was that the group of interviewees was self selecting and small. All but one of the interviewees were aware of WSPs. However, interviews with respondents who were not aware of WSPs would not be very insightful, whereas those who were aware of WSPs had some clear ideas as to what the motivations and barriers were. There was good agreement between the different respondents so although the number is small it is probably representative.

\section{Conclusions}

WSPs have the potential to improve standards and reduce water quality incidents and this was the biggest motivating factor. WSPs form an ideal management and planning tool. WSPs also assist utilities in qualifying for international standards, which is a motivation, although the fact that having implemented a WSP does not in itself quality the supplier for any additional mark or standard may in itself be a barrier. Utilities that care about their image are most likely to implement a WSP. 
The potential for costs savings and NRW reduction is a motivating factor, although there needs to be better communication of successful examples to truly realise this as a motivation. Otherwise the initial investment cost will be perceived as a barrier.

None of the countries in East Africa require WSPs as a regulatory requirement so this is not a motivation in the way that it might be elsewhere. In fact it is a barrier, as either senior managers are not motivated to start implementing a WSP, or utilities are committed to attaining other regulatory requirements and hence struggle to continue to implement their WSPs and expand them into new areas.

The attitude of senior managers can drive or hinder effective WSP implementation as they can ensure that staff prioritise WSPs over other commitments. Smaller or weaker utilities may struggle to implement a WSP as they are constantly engaged in their day-to-day problems and do not have the capacity to plan in the longer term. The lack of support from other stakeholders, particularly on catchment management was also a barrier, as utilities did not believe they could address water safety all the way from the catchment.

While most utilities have sufficient staff to implement a WSP, those staff do not have the relevant skills and there was an almost universal request for more training. There was some criticism of previous international programmes, and an acknowledgement that in the future they need to ensure that the training actually leads to a change in the utility - whilst it is not practical for international agencies to train every staff member, trainees do need to have the capacity to train their peers. Training should not just focus on utilities - there are many other stakeholders whose engagement in WSPs is key for their success, but who may currently be unaware of this initiative. Trainings should also promote simple tools for WSP development and evaluation.

\section{Acknowledgements}


The IWA is acknowledged for its financial support of this research. Tom Williams and Sarah Tibatemwa helped with the research design. Jose Viera, Steve Hrudey and Philip de Souka are also thanked for their contributions to the research.

\section{References}

Bartram, J., Corrales, L., Davison, A., Deere, D., Drury, D., Gordon, B., Howard, G., Rinehold, A. \& Stevens, M. 2009 Water Safety Plan Manual: Step by step risk management for drinking-water suppliers, World Health Organisation, Geneva.

Davison, A., Howard, G., Stevens, M., Callan, P., Fewtrell, L., Deere, D. \& Bartram, J. 2005 Water safety plans, World Health Organisation, Geneva

Dyck, A., Exner, M., \& Kramer, A. 2007 Experimental based experiences with the introduction of a water safety plan for a multi-located university clinic and its efficacy according to WHO recommendations, BMC Public Health 7, 34. DOI: 10.1186/1471-2458-734

Godfrey, S., Chand, C.P., Anward, M., \& Venkataswara Rao, C. 2005 Water-safety plans for piped supplies with limited data - a case study from India, Waterlines 23(4), 19-21. DOI: $10.3362 / 0262-8104.2005 .021$

Gunnarsdottir, M.J. \& Gissurason, L.R. 2008 HACCP and water safety plans in Icelandic water supply: preliminary evaluation of experience, Journal of Water and Health 6, 377-382. DOI: $10.2166 /$ wh.2008.055

Jayaratne, A. 2008 Application of a risk management system to improve drinking water safety. Journal of Water and Health 6, 547-557. DOI: 10.2166/wh.2008.061 
Mahmud, S.G., Shamsuddin, S.A.J., Froze Ahmed, M., Davison, A., Deere, D. \& Howard, G. 2008 Development and implementation of water safety plans for small water supplies in Bangladesh: benefits and lessons learned, Journal of Water and Health 5, 585-597. DOI: 10.2166/wh.2007.045

Mälzer, H.J., Staben, N., Hein, A. \& Merkel, W. 2010 Identification, assessment and control of hazards in water supply: experience form Water Safety Plan implementation in Germany, Water Science and Technology 61, 1307-1315. DOI: 10.2166/wst.2010.026

Rubin, H. J. \& Rubin, I. S. 1995 Qualitative Interviewing: The Art of Hearing Data, Sage Publications, Inc. Thousand Oaks, California

Summerill, C. 2010 Improved Water Safety Planning: Insights into the Role of Organisational Culture. PhD Thesis, Cranfield University.

Summerill, C., Smith, J., Webster, J. \& Pollard, S. 2010 An international review of the challenges associated with securing "buy-in" for water safety plans within providers of drinking water supplies, Journal of Water and Health 8, 387-398. DOI: 10.2166/wh.2010.047

Tibatemwa, S., Godfrey, S., Niwagaba, C. \& Kizito, F. 2005 Implementing water-safety plans in urban piped water supplies in Uganda, Waterlines 23 (4), 8-10. DOI: 10.3362/02628104.2005.017

Viljoen, C. 2010 The World Health Organisation's water safety plan is much more than just an integrated drinking water quality management plan, Water Science and Technology 61, 173-179. DOI: $10.2166 /$ wst.2010.792

WHO 2008 Guidelines for drinking-water quality: incorporating 1st and 2nd addenda, Vol.1, Recommendations. - 3rd edition, World Health Organisation, Geneva. 
Yokoi, H., Embutsu, I., Yoda, M. \& Waseda, K. 2006 Study on the introduction of hazard analysis and critical control point (HACCP) concept of the water quality management in water supply system, Water Science and Technology 52, 483-492.

Preparation:

1) Preliminary actions including team assembly.

System assessment:

2) Document and describe the system.

3) Identify hazards and assess risk.

4) Determine and validate control measures; reassess and prioritise risk.

5) Develop, implement and maintain upgrade/improvement plan.

6) Define monitoring of control measures.

Management and communication:

7) Verify WSP.

8) Prepare management procedures.

9) Develop supporting programmes.

Feedback:

10) Periodic review.

11) Revise the WSP following incident.

Figure 1. Eleven step WSP approach (Bartram et al 2009) 


\begin{tabular}{|c|c|c|c|}
\hline Category & Respondent type & Region of experience & $\begin{array}{l}\text { Number of } \\
\text { respondents }\end{array}$ \\
\hline \multirow[t]{3}{*}{ International Agency } & WOP Co-ordinator & Africa & 3 \\
\hline & UNICEF & Uganda & 1 \\
\hline & Utility & South Africa & 1 \\
\hline \multirow[t]{3}{*}{ Regulator } & Ministry & Uganda & 2 \\
\hline & Regulator & Tanzania & 1 \\
\hline & Water Services Board & Kenya & 2 \\
\hline \multirow[t]{6}{*}{ Utility } & Middle management & Ethiopia & 1 \\
\hline & Senior management & Uganda & 2 \\
\hline & Middle management & Rwanda & 1 \\
\hline & Middle management & Kenya & 3 \\
\hline & Senior management & Kenya & 3 \\
\hline & & Total & 20 \\
\hline
\end{tabular}

Table 1: Interviewees by type 